\title{
Enhanced proliferation and differentiation of Oct4- and Sox2-overexpressing human adipose tissue mesenchymal stem cells
}

\author{
Sei-Myoung Han ${ }^{1}$, Sang-Hun $\mathrm{Han}^{1}$, Ye-Rin $\mathrm{Coh}^{1}$, Goo Jang ${ }^{2}$, Jeong Chan $\mathrm{Ra}^{3}$, Sung-Keun Kang ${ }^{3}$, \\ Hee-Woo Lee ${ }^{4}$ and Hwa-Young Youn ${ }^{1,4}$
}

Mesenchymal stem cells (MSCs) are attractive candidates for clinical repair or regeneration of damaged tissues. Oct4 and Sox2, which are essential transcription factors for pluripotency and self-renewal, are naturally expressed in MSCs at low levels in early passages, and their levels gradually decrease as the passage number increases. Therefore, to improve MSC proliferation and stemness, we introduced human Oct4 and Sox2 for conferring higher expansion and differentiation capabilities. The Oct4-IRES-Sox2 vector was transfected into human adipose tissue MSCs (ATMSCs) by liposomal transfection and used directly. Oct4 and Sox2 were successfully transfected into ATMSCs, and we confirmed maintenance of MSC surface markers without alterations in both red fluorescent protein (RFP) (control) and Oct4/Sox2-ATMSCs. Enhanced proliferative activity of Oct4/Sox2-ATMSCs was shown by WST-1 assay, and this result was further confirmed by cell counting using trypan blue exclusion for a long period. In addition, FACs cell cycle analysis showed that there was a reduction in the fraction of Oct4/Sox2-ATMSCs in G1 with a concomitant increase in the fraction of cells in S, compared with RFP-ATMSCs. Increased levels of cyclin D1 were also seen in Oct4/Sox2-ATMSCs, indicating acceleration in the transition of cells from G1 to S phase. Furthermore, Oct4/Sox2-overexpressing ATMSCs showed higher differentiation abilities for adipocytes or osteoblasts than controls. The markers of adipogenic or osteogenic differentiation were also upregulated by Oct4/Sox 2 overexpression. The improvement in cell proliferation and differentiation using Oct4/Sox2 expression in ATMSCs may be a useful method for expanding the population and increasing the stemness of ATMSCs.

Experimental \& Molecular Medicine (2014) 46, e101; doi:10.1038/emm.2014.28; published online 20 June 2014

Keywords: differentiation; gene engineering; mesenchymal stem cells; Oct4; proliferation; Sox2; stemness

\section{INTRODUCTION}

Mesenchymal stem cells (MSCs) are multipotent cells capable of self-renewal, and they can give rise to a number of unique differentiated mesenchymal cell types. ${ }^{1,2}$ MSCs are attractive candidates for clinical applications for the repair or regeneration of damaged tissues because their isolation is convenient and they lack significant immunogenicity, permitting allogenic transplantation without immunosuppressive drugs. Moreover, there is no ethical controversy on MSCs, and they have the potential to differentiate into tissue-specific cell types. ${ }^{3,4}$ However, the use of MSCs in tissue regeneration has been limited mainly because they have a low proliferation rate and limited lifespan, and they progressively lose their stem cell properties during in vitro expansion.

To induce pluripotent stem cells or to improve the stemness of MSCs, forced expression of pluripotent cell-specific factors (Oct4, Sox2, Nanog and cMyc) or combinations of these genes for reprogramming somatic or adult stem cells $s^{5-9}$ has been shown to induce highly efficient successful reprogramming into pluripotent cells. ${ }^{6}$ Among the four pluripotent factors,

\footnotetext{
${ }^{1}$ Department of Veterinary Internal Medicine, College of Veterinary Medicine, Seoul National University, Seoul, Republic of Korea; ${ }^{2}$ Department of Theriogenology and Biotechnology, College of Veterinary Medicine, Seoul National University, Seoul, Republic of Korea; ${ }^{3}$ Stem Cell Research Center, K-STEMCELL Co. Ltd., Seoul, Republic of Korea and ${ }^{4}$ Research Institute for Veterinary Science, College of Veterinary Medicine, Seoul National University, Seoul, Republic of Korea

Correspondence: Professor HW Lee, Research Institute for Veterinary Science, College of Veterinary Medicine, Seoul National University, Seoul 151-742, Republic of Korea.

E-mail: Ihwdinks90@gmail.com

or Professor HY Youn, Department of Veterinary Internal Medicine, College of Veterinary Medicine, Seoul National University, Seoul 151-742, Republic of Korea.

E-mail: hyyoun@snu.ac.kr

Received 26 July 2013; revised 11 November 2013; accepted 10 February 2014
} 
Oct 4 and Sox 2 are transcription factors essential to pluripotent and self-renewing phenotypes. ${ }^{10,11}$ It is well known that Oct4 is a key transcription factor essential for self-renewal and survival of MSCs, ${ }^{7,8,12}$ and it has a unique role in the development and determination of pluripotency. This gene constitutes the core regulatory network that suppresses differentiation-associated genes, thereby maintaining pluripotency of the cells. ${ }^{13}$ Sox 2 has a critical role in the maintenance of embryonic and neural stem cells and holds great promise in research involving induced pluripotency. Furthermore, Go et al. ${ }^{5}$ observed that Sox 2 is not only essential for pluripotency and self-renewal of embryonic stem cells, but is also expressed in somatic stem cells with superior expansion and differentiation potential.

Both Oct4 and Sox2 are naturally expressed in both embryonic stem cells and adult stem cells. However, they are usually expressed at low levels in early-passage MSCs and gradually decrease as the passage number increases. ${ }^{6,7}$ Several researchers have studied the effect of Oct4 or Sox2 overexpression in MSCs, ${ }^{5,6,9,14}$ but the results are still debatable. Although their roles are not clearly identified in MSCs, it seems apparent that Oct4 and Sox2 have reciprocal actions. ${ }^{15-17}$ However, the effect of co-expression of Oct4 and Sox2 in MSCs has not been investigated. In this study, we co-expressed Oct 4 and Sox 2 with the aim of conferring higher capabilities for proliferation and differentiation.

\section{MATERIALS AND METHODS}

\section{Cell cultures}

Human adipose tissues were obtained by simple liposuction of abdominal subcutaneous fat from a donor after obtaining informed consent according to the guidelines of the Institutional Review Board (IRB) of the K-STEMCELL, Seoul, Korea. Cells were maintained in RKCM (MSC proliferation media, K-STEMCELL) at subconfluent levels to prevent spontaneous differentiation.

\section{Generation of Oct4/Sox2-overexpressing human ATMSCs} Oct4-IRES-Sox2 was inserted into the PB-CA vector (Addgene, Cambridge, MA, USA). Human adipose tissue MSCs (ATMSCs) were then transfected with the Oct4-IRES-Sox2 vector using D-fection transfection reagent (Lugen Sci, Seoul, Korea) according to the manufacturer's instructions. Oct4/Sox2-containing plasmid and $\mathrm{D}$-fection complex were added to the cells in the appropriate volume of ATMSC culture medium. Cells were fed with fresh culture medium at $24 \mathrm{~h}$ after transfection. Transfected cells were frozen at either P4 or P5 to make cryopreserved cell stocks. For cellular analyses, we used the transfected cells directly (P4 or P5), or thawed the cryopreserved cells. Red fluorescent protein (RFP) inserted into the PB-CA vector was used as a control, and it was generated using the same methods.

\section{Flow cytometric analysis of cell surface marker expression in ATMSCs}

To analyze the cell surface marker expression in RFP- and Oct4/Sox2ATMSCs, flow cytometry was performed. By passage 5, a homogenous population of rapidly dividing cells with fibroblastoid morphology was obtained. ATMSCs were fixed with $70 \%$ ethanol at $4{ }^{\circ} \mathrm{C}$ and stained for $30 \mathrm{~min}$ on ice with primary antibodies that recognize various surface molecules. Phycoerythrin-conjugated (PE) mouse anti-human CD29 (BD Biosciences, San Jose, CA, USA), FITC (fluorescein isothiocyanate-conjugated) mouse anti-human CD31 (BD Biosciences), PE mouse anti-human CD34 (BD Biosciences), FITC mouse anti-human CD44 (BD Biosciences), FITC mouse anti-human CD45 (BD Biosciences), PE mouse anti-human CD73 (BD Biosciences Pharmingen, San Diego, CA, USA), PE mouse antihuman CD90 (BD Biosciences), and PE anti-human CD105/endoglin (R\&D system, Minneapolis, MN, USA) were used for cell surface antigen detection. The immunophenotype of MSCs was analyzed using a FACS Calibur flow cytometer (BD Biosciences, Bedford, MA, USA) with CELLQuest software (BD Biosciences).

\section{RT-PCR analysis}

Total RNA was isolated from RFP- or Oct4/Sox2-transfected ATMSCs using Trizol (Invitrogen, Carlsbad, CA, USA) following the manufacturer's protocol. Briefly, samples were transferred to a tube containing $1 \mathrm{ml}$ of RNA extraction solution. The homogenate was then extracted with chloroform, precipitated using isopropanol, washed with ethanol and then resuspended in $30 \mu \mathrm{l}$ distilled water. RNA concentration and purity were determined using a Nanophotometer (Implen, Munich, Germany) at 260 and $280 \mathrm{~nm}$. Samples exhibited an absorbance ratio (260/280) of $\geqslant 1.8$. The first-strand cDNA was obtained by reverse transcription using $3 \mu \mathrm{g}$ total RNA, M-MuLV RT and an oligo(dT)-18 primer, according to the manufacturer's instructions (Invitrogen). Primer sequences are shown in Table 1. PCR products were electrophoresed on $1.5 \%$ agarose gels to verify DNA fragment sizes.

\section{Cell proliferation analysis}

To determine the effects of Oct4/Sox 2 overexpression on MSC proliferation rates, cell proliferation and viability were evaluated by WST-1 cell proliferation and trypan blue exclusion assays. For the WST-1 cell proliferation assay, cells were seeded in 96-well plates at $5 \times 10^{3}$ cells/well. Each cell was assayed in triplicate, including RFP-ATMSCs wells. Cells were then incubated for 4 (day 0), 24 (day 1), 48 (day 2) and $72 \mathrm{~h}$ (day 3), and then $10 \mu \mathrm{l}$ WST- 1 reagent was added to each well. The reaction proceeded for $2 \mathrm{~h}$ at $37^{\circ} \mathrm{C}$ with $5 \% \mathrm{CO}_{2}$. The absorbance of the samples at $450 \mathrm{~nm}$ was measured using a microplate reader.

For trypan blue exclusion, ATMSCs were seeded into six-well culture plates at $1 \times 10^{5}$ cells/well in duplicate and then incubated at $37^{\circ} \mathrm{C}$ with $5 \% \mathrm{CO}_{2}$. After day 1, 2, 3, 5, 7 and 9, cells were collected and stained with $0.4 \%$ trypan blue solution. Enumeration of viable cells was carried out under a phase contrast microscope with a hemocytometer.

\section{Cell cycle analysis}

Three days after transfection, ATMSCs were washed twice with PBS and harvested by trypsinization. The cells were then washed again with PBS and fixed with $70 \%$ ethanol at $-20^{\circ} \mathrm{C}$ for 1 day. The fixed cells were washed with ice-cold PBS and stained with $50 \mu \mathrm{g} \mathrm{ml}^{-1}$ propidium iodide (Sigma, St. Louis, MO, USA) in the presence of $100 \mu \mathrm{g} \mathrm{ml}^{-1}$ RNase A (Sigma) for $30 \mathrm{~min}$. The cell cycle was analyzed using a FACS Calibur flow cytometer (BD Biosciences).

\section{Western blot analysis}

After RFP- and Oct4/Sox2-ATMSCs were collected by centrifugation, the pellet was resuspended in lysis buffer ( $25 \mathrm{~mm}$ Tris, $\mathrm{pH} 7.5,150 \mathrm{~mm}$ $\mathrm{NaCl}, 1 \% \mathrm{NP}-40,0.5 \%$ sodium deoxycholate and $0.1 \%$ SDS) containing proteinase inhibitors and incubated on ice for $30 \mathrm{~min}$. Following centrifugation at $16000 \times \mathrm{g}$ for $15 \mathrm{~min}$ at $4{ }^{\circ} \mathrm{C}$, the supernatant 
Table 1 Primer sequences for RT-PCR amplification of target genes

\begin{tabular}{|c|c|c|c|}
\hline Gene & Primer & Gene Bank accession number & Product size (bp) \\
\hline \multirow[t]{2}{*}{ Oct4 } & F: 5'-TGCAGAAAGAACTCGAGCAA-3' & NM_002701.4 & 1082 \\
\hline & R: 5'-ACACTCGGACCACATCCTTC-3' & & \\
\hline Sox2 & R: 5'-ATGTAGGTCTGCGAGCTGGT-3' & & \\
\hline PPAR $\gamma$ & F: 5'-AAGACCACTCCCACTCCTTTG-3' & NM_005037.5 & 554 \\
\hline LPL & R: 5'-CTGAAGACACAGCTGAGGAC-3' & & \\
\hline \multirow[t]{2}{*}{ Osteocalcin } & F: 5'-GTGCAGAGTCCAGCAAAGGT-3' & NM_199173.4 & 175 \\
\hline & R: 5'-TCAGCCAACTCGTCACAGTC-3' & & \\
\hline \multirow[t]{2}{*}{ Collagen I } & F: 5'-GTCGAGGGCCAAGACGAAG-3' & JQ236861.1 & 133 \\
\hline & R: 5'-CAGATCACGTCATCGCACAA-3' & & \\
\hline GAPDH & F: 5'-AAGTGGATATTGTTGCCATC-3' & NM_002046.4 & 454 \\
\hline
\end{tabular}

Abbreviations: LPL, lipoprotein lipase; PPAR $\gamma$, peroxisome proliferator-activated receptor gamma.
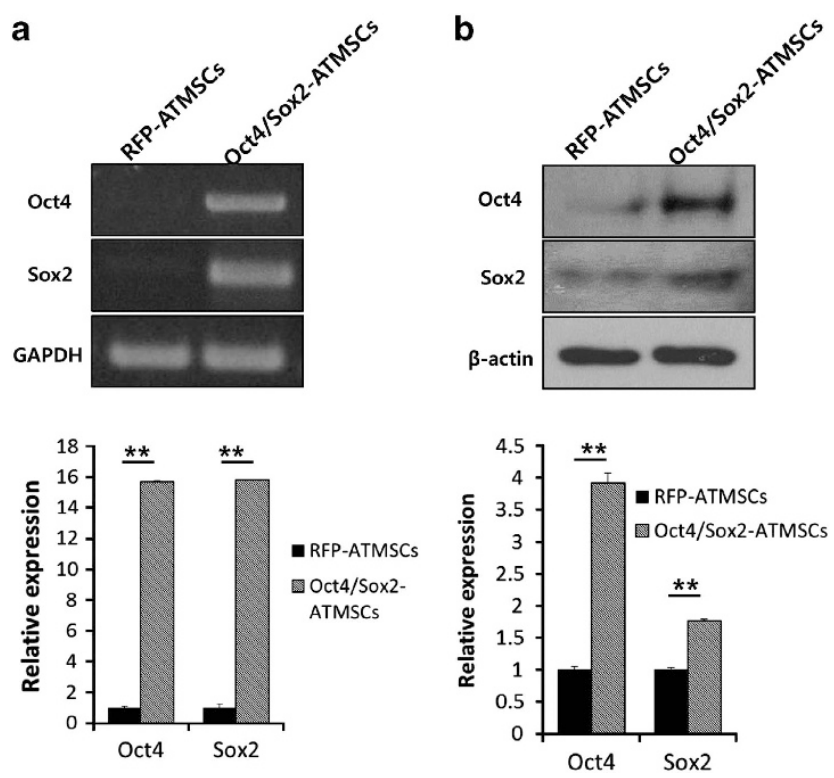

Figure 1 Expression analysis of Oct4 and Sox2 in Oct4/Sox2ATMSCs. (a) In RT-PCR analysis, the mRNA expression levels of Oct4 and Sox2 in Oct4/Sox2-ATMSCs were significantly higher than those of RFP-ATMSCs at $24 \mathrm{~h}$ post-transfection. Band densities in RT-PCR were evaluated semi-quantitatively by densitometry. Results are the ratio of Oct4 and Sox2 expression normalized to GAPDH mRNA levels. (b) Western blots show high levels of Oct4 and Sox2 expression in Oct4/Sox2-ATMSCs. Band densities in western blot analysis were evaluated semiquantitatively by densitometry. Results are the ratio of Oct4 and Sox2 expression normalized to beta-actin protein levels. Data are representative of three independent experiments, with similar results. Data are expressed as the means + s.d. ${ }^{*} P<0.01$ compared with the control (RFP-ATMSCs) value as determined by Student's $t$-test. containing the total cell extract was collected and kept at $-80{ }^{\circ} \mathrm{C}$. Protein from the cell extracts were electrophoresed and transferred onto a Hybond-PVDF membrane (Amersham Biosciences, Piscataway, NJ, USA). The membrane was incubated for $1 \mathrm{~h}$ at room temperature in blocking buffer (TBS-T containing 5\% skim milk) to block nonspecific protein binding and then incubated overnight at $4{ }^{\circ} \mathrm{C}$ with a primary antibody against Oct4 (1:1000), Sox2 (1:500), cyclin D1 (1:1000) or $\beta$-actin (1:1000) (Santa Cruz Biotechnology Inc., Santa Cruz, CA, USA) diluted in blocking buffer. The membrane was washed with TBS-T and incubated with secondary antibody diluted (1:3000) in blocking buffer for $1 \mathrm{~h}$ at room temperature. Antibody binding was visualized with a West-Q Chemiluminescent Substrate Plus Kit (GenDEPOT, Barker, TX, USA).

\section{Osteogenic and adipogenic differentiation}

Human RFP or Oct4/Sox2-ATMSCs that were $\sim 80 \%$ confluent were induced to differentiate into adipocytes or osteocytes for 21 days in adipogenic or osteogenic media, respectively.

To induce adipogenic differentiation, both RFP- and Oct4/Sox2-

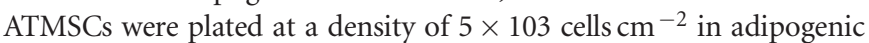
induction medium (Gibco, Grand Island, NY, USA). The medium was changed every 3 days. After 7, 14 and 21 days, cells were fixed with 4\% paraformaldehyde and oil red $\mathrm{O}$ staining was conducted to identify lipid droplets.

To induce osteogenic differentiation, both RFP- and Oct4/Sox2ATMSCs were plated at a density of $5 \times 103$ cells cm $^{-2}$ (Gibco). The medium was changed every 3 days. After 7, 14 and 21 days, cells were fixed with $4 \%$ paraformaldehyde and alizarin red S staining was performed to assess the mineralized matrix content.

\section{Statistical analysis}

All experiments were performed at least twice. Values are expressed as mean + standard deviation. The probability associated with a Student's $t$-test was performed for comparison of the parameters between two groups. $P$-values $<0.05$ were considered statistically significant. 

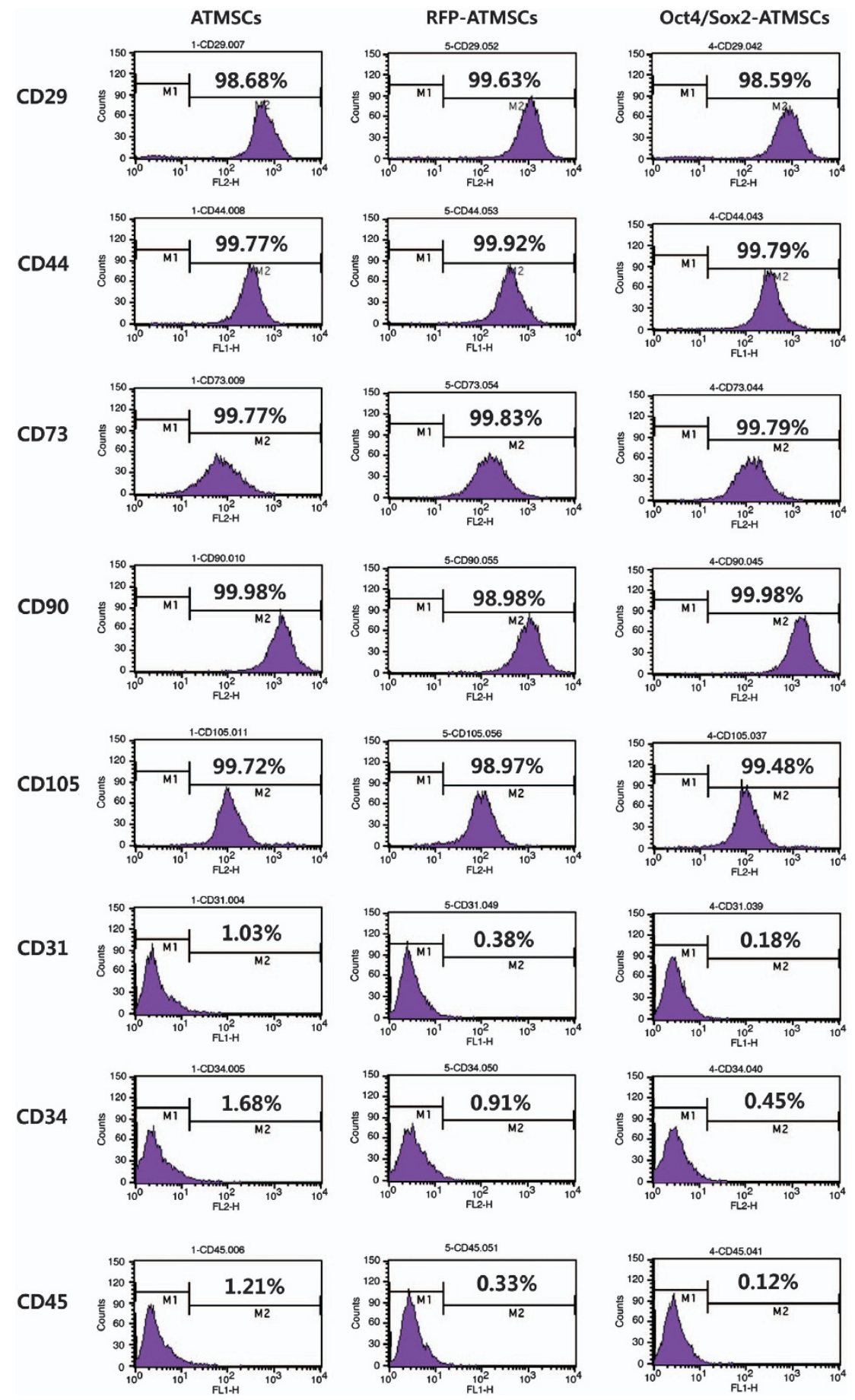

Figure 2 Immunophenotyping of RFP- and Oct4/Sox2-transfected ATMSCs. Non-transfected MSCs at passage 3, RFP-transfected ATMSCs at passage 5 and Oct4/Sox2-transfected ATMSCs at passage 5 were immunophenotyped for CD29, CD31, CD34, CD44, CD45, CD73, CD90 and CD105 by flow cytometry. The expression of surface markers of ATMSCs transduced with Oct4/Sox2 or RFP were not different from those of non-transfected ATMSCs. Data are representative of three independent experiments, with similar results.

\section{RESULTS}

Expression analysis of Oct4 and Sox2 in ATMSCs transfected with Oct4/Sox 2 genes

To assess Oct4 and Sox2 expression in ATMSCs transfected with Oct4/Sox2 genes (Oct4/Sox2-ATMSCs), we performed RT-PCR and western blot analysis (Figure 1). The levels of Oct4 and Sox2 mRNA were significantly higher in ATMSCs than in RFP-ATMSCs, whereas the expression levels of Oct4 and Sox2 in RFP-ATMSCs were almost undetectable. Concurrently, the western blot analysis results revealed that the expression of Oct4 and Sox 2 protein was significantly upregulated in Oct4/Sox2-ATMSCs. These results showed that Oct4/Sox2-ATMSCs were successfully generated by liposomal transfection. 


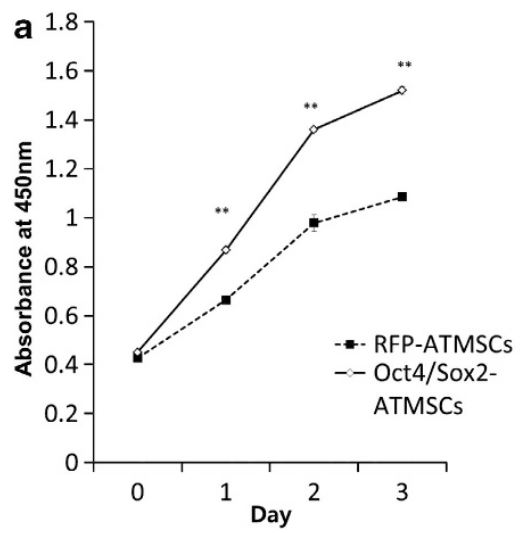

\section{b}

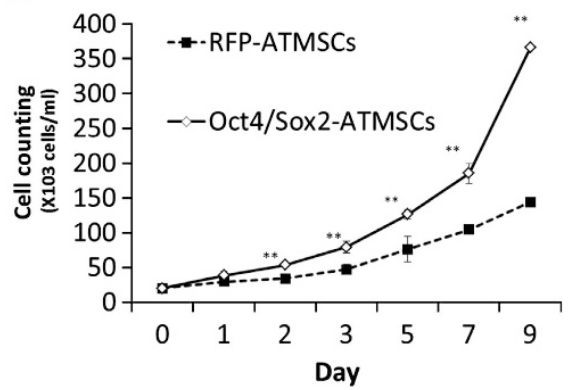

Figure 3 Proliferation assay using Oct4/Sox2-ATMSCs. (a) WST-1 assay showed that Oct4/Sox2-ATMSCs have higher cell metabolic activity than RFP-ATMSCs at 1, 2 and 3 days. (b) In the trypan blue exclusion assay, viable cell numbers were increased significantly in Oct4/Sox2-ATMSCs cultured for 9 days compared with RFP-ATMSCs. Data are expressed as the mean \pm s.d. ${ }^{* *} P<0.01$ compared with the corresponding control value as determined by Student's $t$-test.

a
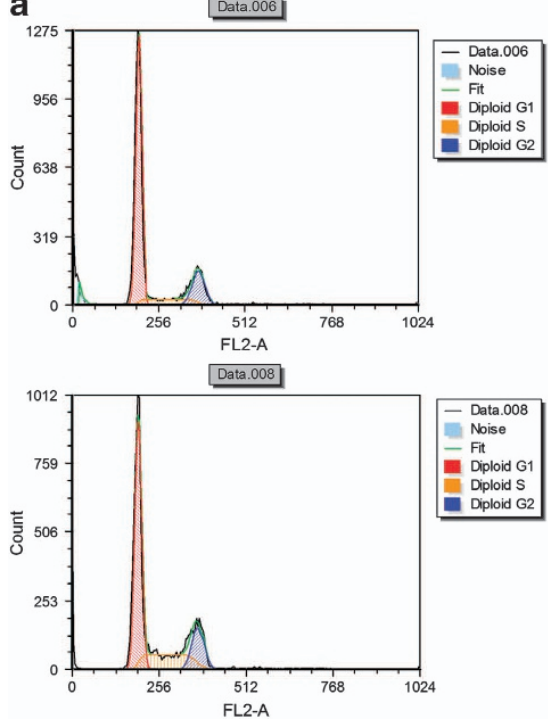

b

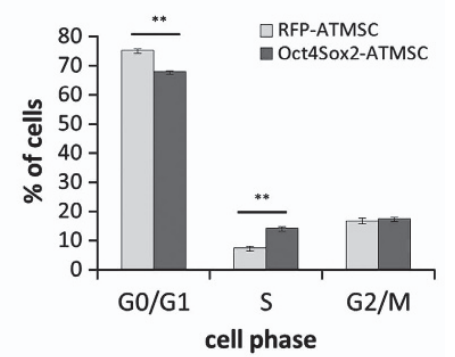

c

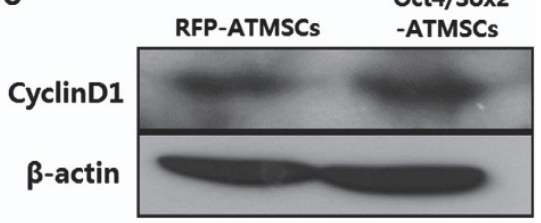

Figure 4 Cell cycle analysis by flow cytometry, and western blot analysis of cyclin D1 in Oct4/Sox2-ATMSCs. (a) Representative flow cytometry results of RFP- and Oct4/Sox2-ATMSCs (b) The percentage of cells at each cell cycle stage as determined by flow cytometry analysis. Compared with the control (RFP-ATMSCs), cell cycle analysis showed a decrease in the proportion of Oct4/Sox2-ATMSCs in the G0/G1 phase, whereas an increase was observed in the proportion of those in the S phase. (c) The expression of cyclin D1, which is a major regulator, was increased in Oct4/Sox2-ATMSCs compared with RFP-ATMSCs. Data are expressed as the mean $+\mathrm{s} . \mathrm{d}$. ${ }^{* * P} P 0.01$ compared with the corresponding control value as determined by Student's $t$-test.

Immunophenotyping of both RFP- and Oct4/Sox2-ATMSCs The surface markers CD29, CD44, CD73, CD90, CD105, CD31, CD34 and CD45 were used to evaluate whether the immunophenotypic characteristics of ATMSCs changed with gene transfection at passage 5. Flow cytometry analysis showed high expression of CD29, CD44, CD73, CD90 and CD105, and the absence of CD31, CD34 and CD45 surface markers on both RFP- and Oct4/Sox2-transfected ATMSCs (Figure 2). There were no differences in expression of surface markers in ATMSCs with both gene modifications. The result of flow cytometric analyses indicates that the expression of ATMSC surface markers is not changed by Oct4/Sox 2 gene transfection.

\section{Enhanced proliferative potential of Oct4/Sox2-ATMSCs}

To assess the proliferative ability of Oct4/Sox2-ATMSCs, we examined cell growth by WST-1 assay, which measures cell viability relative to the metabolic activity (Figure $3 \mathrm{a}$ ). The Oct4/Sox2 overexpression in ATMSCs resulted in a timedependent increase in proliferation. This result was further confirmed by trypan blue exclusion assay (Figure 3b), which serves as an index of cell viability. It was apparent that the number of viable Oct4/Sox2-ATMSCs was increased significantly compared with that of RFP-ATMSCs. The data demonstrate that Oct4/Sox2-expressing ATMSCs have much higher expansion potential and cell viability than control cells (RFP-ATMSCs). 


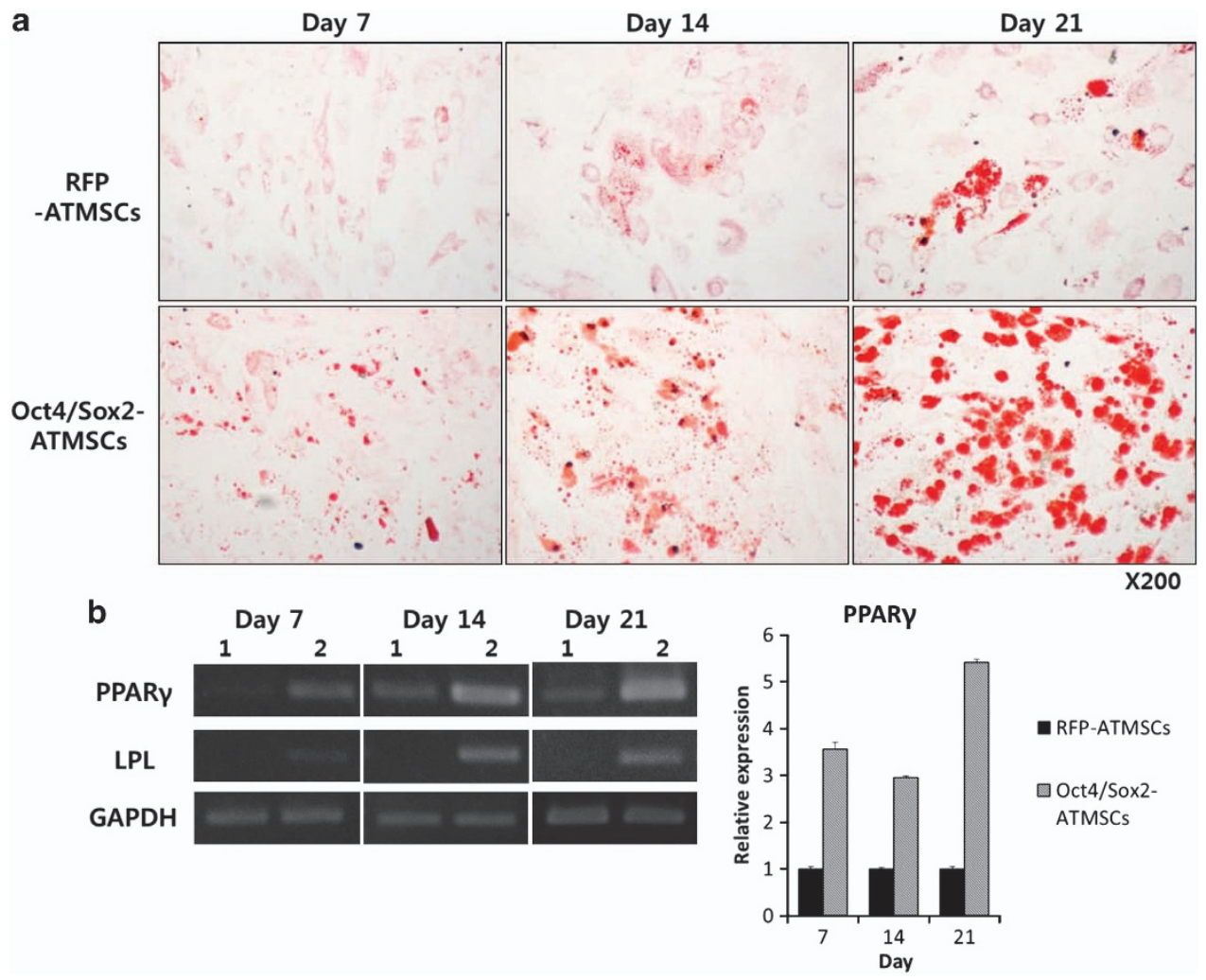

Figure 5 Adipogenic differentiation assay using Oct4/Sox2-ATMSCs and RT-PCR analysis for adipogenic markers. (a) Oil red 0 staining for lipid droplets in Oct4/Sox2-transfected ATMSCs was strong at 7, 14 and 21 days during adipogenic differentiation compared with RFP-ATMSCs. (b) RT-PCR results show a significant increase in PPAR $\gamma$ and lipoprotein lipase mRNA expression at 7, 14 and 21 days during adipogenic differentiation. Band densities of PPAR $\gamma$ in RT-PCR were evaluated semi-quantitatively by densitometry. Results are the ratio of PPAR $\gamma$ expression normalized to GAPDH mRNA levels. Data are representative of three independent experiments with similar results. (1) RFP-ATMSCs, (2) Oct4/Sox2-ATMSCs.

\section{Acceleration of the G1 to $S$ phase transition in Oct4/Sox2-ATMSCs}

We evaluated the effects of Oct $4 /$ Sox 2 overexpression on the cell cycle (Figure 4a) based on our previous finding that Oct4/Sox2 overexpression promotes cell proliferation. Cell cycle analysis by flow cytometry showed that in Oct4/Sox2ATMSCs, $67.99 \pm 0.69 \%$ of cells were in the G0/G1 phase, $14.38 \pm 0.84 \%$ were in the $S$ phase and $17.65 \pm 1.36 \%$ were in the G2/M phase. However, the percentage of $\mathrm{S}$ and $\mathrm{G} 2 / \mathrm{M}$ phase cells in RFP-ATMSCs was much lower $(7.59 \pm 0.62 \%$, $16.73 \pm 0.93 \%)$. All Oct $4 /$ Sox 2 -ATMSCs had a reduction in the fraction of cells in G1 with a concomitant increase in the fraction of cells in S compared with RFP-ATMSCs.

To verify cell cycle progression, we assessed the changes in the protein levels of a major regulator, cyclin D1, which promotes G1-to-S-phase transition and cell proliferation. As shown in Figure 4b, Oct4/Sox2 upregulation in ATMSCs resulted in increased cyclin D1 expression. These data show that Oct4/ Sox2 overexpression promotes ATMSC proliferation, at least in part, by promoting the transition of cells from G1 to S phase.

\section{Improved adipogenic and osteogenic differentiation of Oct4/Sox2-ATMSCs}

To study the effect of Oct $4 / \mathrm{Sox} 2$ overexpression on the pluripotency of ATMSCs, we examined the differentiation potential of Oct4/Sox2-ATMSCs into adipocytes and osteoblasts. We assessed the differentiation potency by comparing the staining intensity and the expression of fat and bone genes at the transcriptional level. The staining intensity of oil red $\mathrm{O}$ for lipid droplets in Oct4/Sox2-ATMSCs was stronger than that in RFP-ATMSCs, suggesting that Oct4/Sox2 expression promotes adipogenesis of ATMSCs (Figure 5a). To validate these results, we analyzed lineage-specific gene expression. Peroxisome proliferator-activated receptor gamma (PPAR $\gamma)$ and lipoprotein lipase, genetic markers of adipose tissue, were upregulated after Oct4/Sox2 overexpression (Figure 5b). Similarly, alizarin red $\mathrm{S}$ staining intensity was much stronger in Oct4/Sox2-ATMSCs than in RFP-ATMSCs (Figure 6a). Consistent with these phenotypic results, markers of osteogenic differentiation (collagen I and osteocalcin) were upregulated by Oct4/Sox2 gene engineering (Figure 6b). The results of the gene expression patterns and phenotypic analyses indicate that Oct $4 /$ Sox 2 overexpression significantly promotes the ability of ATMSCs to differentiate into adipocytes and osteoblasts.

\section{DISCUSSION}

In the present study, we successfully engineered ATMSCs to overexpress Oct 4 and Sox 2 by using a PB-CA vector, which has 
a
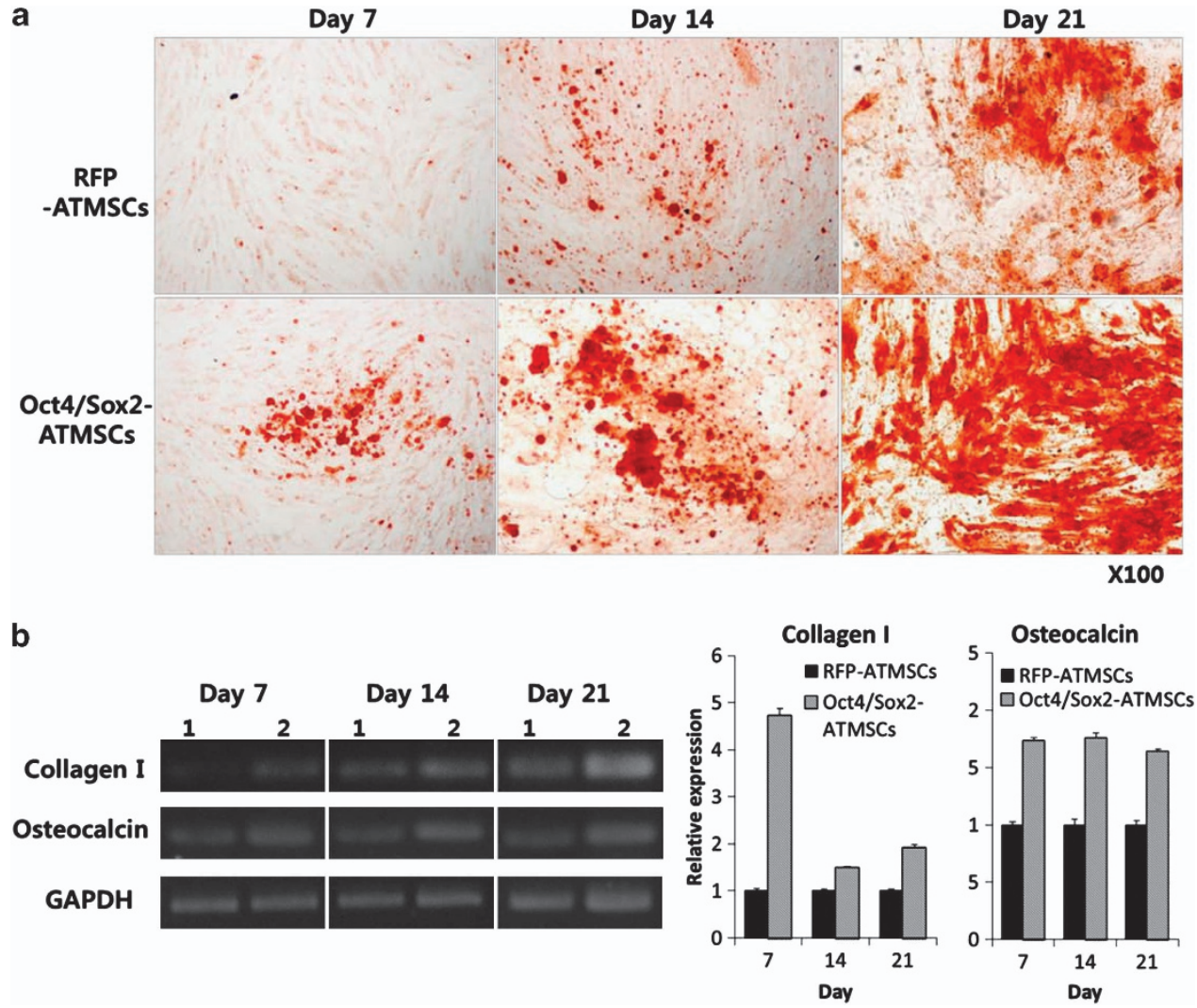

Figure 6 Osteogenic differentiation assay using Oct4/Sox2-ATMSCs and RT-PCR analysis for osteogenic markers. (a) Alizarin red S staining for mineralization in Oct4/Sox2-ATMSCs was strong at 7, 14 and 21 days during osteogenic differentiation compared with RFP-ATMSCs. (b) RT-PCR results show upregulation of collagen I and osteocalcin mRNA levels in Oct4/Sox2-ATMSCs at 7, 14 and 21 days during osteogenic differentiation compared with RFP-ATMSCs. Band densities of collagen I and osteocalcin in RT-PCR were evaluated semi-quantitatively by densitometry. Results are the ratio of collagen I and osteocalcin expression normalized to GAPDH mRNA levels. Data are representative of three independent experiments with similar results. (1) RFP-ATMSCs, (2) Oct4/Sox2-ATMSCs.

the advantage of integration into the host cell genome. The $\mathrm{PB}-\mathrm{CA}$ vector backbone requires the PBase vector to 'piggyBac transpose' and has a high copy number. After $24 \mathrm{~h}$ transfection with Oct4/Sox2 in ATMSCs, Oct4/Sox2-ATMSCs appear to show no differences in cell morphology compared with non-transfected ATMSCs although a low level of cell death was observed in the liposomally transfected cells. RT-PCR demonstrated high efficiency of exogenous Oct4 and Sox2 production, and high Oct4 and Sox 2 protein levels were detected by western blot. In addition, immunophenotyping by flow cytometric analysis showed that the genetic modification of ATMSCs with Oct4/Sox2 did not alter MSC surface markers.

The results of WST-1 and trypan blue exclusion assays showed that Oct4/Sox2-overexpressing cells had significantly higher viability and proliferation rates than control cells. These results were further evaluated in cell cycle progression by cell cycle analysis and western blot analysis of cyclin D1. The cell cycle is a series of events that occurs in a cell leading to its division and replication, and is a critical process for determining cell proliferation and senescence. ${ }^{6}$ The G0/G1 phase is the non-proliferative or resting phase; the $S$ phase involves DNA replication; in G2, the cell is ready for division; and in the $\mathrm{M}$ phase, mitosis occurs. In this study, Oct4/Sox 2 overexpression in ATMSCs resulted in an extended $\mathrm{S}$ phase. Cell cycle progression is tightly regulated by cyclins, and cyclin D1 is the first cyclin produced in the cell cycle. Upregulation of cyclin $D 1$ can cause premature entrance into the $S$ phase, indicating a rate-limiting role for cyclin $\mathrm{D} 1$ in the $\mathrm{G} / \mathrm{S}$ transition. ${ }^{18,19}$ The expression of cyclin D1 influences cell cycle progression and consequently, stem cell proliferation. Taken together, these results indicate that Oct4/Sox 2 overexpression causes high enough levels of cyclin D1 for the cell to initiate DNA replication and be in the extended $S$ phase, resulting in cell growth and proliferation. Thus, these observations appear to indicate a stronger effect of Oct $4 /$ Sox 2 expression on cell proliferation compared with control cells, at least in part, by accelerating the transition of cells from G1 to $S$ phase.

We showed that Oct4/Sox 2 overexpression increased the number of positive cells stained with oil red $\mathrm{O}$ or alizarin red $\mathrm{S}$ and the expression of adipogenic or osteogenic markers, indicating an enhanced capacity of Oct4/Sox2-ATMSCs for differentiation towards adipogenesis or osteogenesis. This result means that the overexpression of Oct4 and Sox2 increased differentiation ability in the prepared differentiation media containing all reagents required for inducing MSCs to 
be committed to the adipogenesis and osteogenesis pathway. The effect of Oct 4 and Sox 2 on the differentiation mechanism of stem cells was studied by other some researchers. Ben-Shushan et al. ${ }^{20}$ reported that the down regulation of Zfp42 mRNA (preventing differentiation) caused by induction of elevated Oct-4 is consistent with the finding that overexpression of Oct- 4 represses activation of the Zfp42 promoter mediated via Rox-1. The increasing the levels of Sox2, and thus limiting the expression of several Sox2:Oct-4 target genes (Oct4, Nanog, FGF-4, UTF-1, Lefty-1 and BMP4) that are critical for stem cell pluripotency, may trigger the differentiation of cells.

As Oct4 and Sox2 are important at the transcriptional level to maintain pluripotency in embryonic stem cells, ${ }^{10,11,21}$ their expression level is important within the framework of stem cell differentiation. Thus, it is well known that Oct4 levels govern the balance between differentiation and de-differentiation and that maintaining Oct4 expression within a certain range appears to be critical for stem cell renewal, with any increase or decrease triggering differentiation to endoderm/mesoderm or trophectoderm, respectively. ${ }^{22}$ In contrast with Oct4, overexpression of Sox 2 promotes embryonic stem cells to differentiate into cells that express a wide range of markers associated with neuroectoderm and mesoderm, as well as markers for trophectoderm cells. ${ }^{23}$ It has been reported that increase of twofold or less in the levels of Sox 2 triggers cell differentiation. On the basis of these studies, a proper level of overexpression of these genes is crucial for inducing stimulation of the cell stemness. Our results showing that co-overexpression of Oct4 and Sox2 genes effectively enhanced mesodermal differentiation potency indicates increased stemness of ATMSCs.

In MSC research, studies on overexpression of pluripotent genes in MSCs are not well identified and are still controversial. Lim et al. ${ }^{7}$ established stable Oct4-overexpressing MSCs and showed that this promoted cell proliferation and improved adipogenesis and chondrogenesis. Conversely, Nanog overexpression has been shown to slow down adipogenesis of MSCs. In other studies, increases in both Nanog and Oct4 increased proliferation and trilineage differentiation potential but decreased spontaneous differentiation, suggesting that Oct4 and Nanog are not only essential for the maintenance of pluripotency in embryonic stem cells but also for maintaining MSC properties. ${ }^{24}$ However, Jaganathan and Bonnet ${ }^{14}$ reported that exogenous Oct4 induced early senescence, as evidenced by increased expression of p16INK4a and p14, which are key regulators of cellular senescence in MSCs. Sox2 is also important for maintaining proliferation and osteogenic differentiation potentials of $\mathrm{MSCs}^{5}{ }^{5}$ and replicative senescence and multipotentiality of MSCs during in vitro expansion may be regulated predominantly through Sox $2 .^{6}$ Although their roles in MSCs are still controversial, Oct4 and Sox2 have been described previously to drive pluripotent-specific expression of a number of genes through cooperative interaction. ${ }^{25}$ Chew et al. ${ }^{16}$ showed that Oct4 and Sox 2 , which bind independently to their respective binding motifs, act synergistically to activate these enhancers. Furthermore, the Oct-Sox enhancers regulate the expression of pluripotent stem cell-specific genes, including Nanog, Oct4 and Sox2 itself, suggesting that this enhancer is at the top of the pluripotent cell genetic regulatory network. ${ }^{15}$ For these reasons, the study of Oct4 and Sox2 has provided major insights into the transcriptional circuitry responsible for sustaining the self-renewal and pluripotency of embryonic stem cells. Therefore, we selected these two transcriptional genes for genetic modification of ATMSCs and showed that Oct 4 and Sox 2 overexpression significantly affect the cell viability and stemness of ATMSCs in an in vitro culture system.

In the advancement of stem cell therapy, the acquisition of clinically relevant numbers of stem cells in a timely manner and the maintenance of stem cell properties are challenging issues. ${ }^{26,27}$ In this context, MSCs transduced with Oct4/Sox2 have useful clinical applications because they have short culture duration, and costs and labor are reduced. Furthermore, Oct4 and Sox2 improved adipogenic and osteogenic differentiation, which may have resulted from enhancement of ATMSC stemness. Multilineage capacity and proliferation in vitro were interpreted at the time as indicative of in vivo multipotency and self-renewal properties-the hallmarks of 'stemness. ${ }^{28}$ In conclusion, our data suggest that Oct4/Sox 2 gene engineering could be a useful method for gaining high-quality ATMSCs with shortened culture time and the ability to meet efficacy endpoints in clinical studies of MSC-based tissue engineering.

\section{CONFLICT OF INTEREST}

The authors declare no conflict of interest.

\section{ACKNOWLEDGEMENTS}

This research was supported by the Basic Science Research Program through the National Research Foundation of Korea (NRF) funded by the Ministry of Education, Science and Technology

(2012R1A1A2007693). This study was partially supported by the Research Institute for Veterinary Science and BK21 Plus Program for Veterinary Science, Seoul National University. We also thank Stem Cell Research Center of K-STEMCELL Co. Ltd.

1 da Silva ML, Chagastelles PC, Nardi NB. Mesenchymal stem cells reside in virtually all post-natal organs and tissues. J Cell Sci 2006; 119: 2204-2213.

2 Schäffler A, Büchler C. Concise review: adipose tissue-derived stromal cells-basic and clinical implications for novel cell-based therapies. Stem Cells 2007; 25: 818-827.

3 Sasaki M, Abe R, Fujita Y, Ando S, Inokuma D, Shimizu H. Mesenchymal stem cells are recruited into wounded skin and contribute to wound repair by transdifferentiation into multiple skin cell type. J Immun 2008; 180: 2581-2587.

4 Toma C, Pittenger MF, Cahill KS, Byrne BJ, Kessler PD. Human mesenchymal stem cells differentiate to a cardiomyocyte phenotype in the adult murine heart. Circulation 2002; 105: 93-98.

5 Go MJ, Takenaka C, Ohgushi H. Forced expression of Sox2 or Nanog in human bone marrow derived mesenchymal stem cells maintains their expansion and differentiation capabilities. Exp Cell Res 2008; 314: 1147-1154. 
6 Yoon D, Kim Y, Jung H, Paik S, Lee J. Importance of Sox2 in maintenance of cell proliferation and multipotency of mesenchymal stem cells in low-density culture. Cell Prolif 2011; 44: 428-440.

7 Liu TM, Wu YN, Guo XM, Hui JHP, Lee EH, Lim B. Effects of ectopic Nanog and Oct4 overexpression on mesenchymal stem cells. Stem Cells Dev 2009; 18: 1013-1022.

8 Kim JB, Sebastiano V, Wu G, Araúzo-Bravo MJ, Sasse P, Gentile L et al. Oct4-induced pluripotency in adult neural stem cells. Cell 2009; 136: 411-419.

9 Seo K-W, Lee S-R, Bhandari DR, Roh K-H, Park S-B, So A-Y et al. OCT4A contributes to the stemness and multi-potency of human umbilical cord blood-derived multipotent stem cells (hUCB-MSCs). Biochem Biophys Res Commun 2009; 384: 120-125.

10 Nichols J, Zevnik B, Anastassiadis K, Niwa H, Klewe-Nebenius D, Chambers I et al. Formation of pluripotent stem cells in the mammalian embryo depends on the POU transcription factor Oct4. Cell 1998; 95: 379-391.

11 Avilion AA, Nicolis SK, Pevny LH, Perez L, Vivian N, Lovell-Badge R. Multipotent cell lineages in early mouse development depend on SOX2 function. Genes Dev 2003; 17: 126-140.

12 Pesce M, Schöler HR. Oct-4: gatekeeper in the beginnings of mammalian development. Stem Cells 2001; 19: 271-278.

13 Boyer LA, Lee TI, Cole MF, Johnstone SE, Levine SS, Zucker JP et al. Core transcriptional regulatory circuitry in human embryonic stem cells. Cell 2005; 122: 947-956.

14 Jaganathan BG, Bonnet D. Human mesenchymal stromal cells senesce with exogenous OCT4. Cytotherapy 2012; 14: 1054-1063.

15 Rodda DJ, Chew J-L, Lim L-H, Loh Y-H, Wang B, Ng H-H et al. Transcriptional regulation of nanog by OCT4 and SOX2. J Biol Chem 2005; 280: 24731-24737.

16 Chew J-L, Loh Y-H, Zhang W, Chen X, Tam W-L, Yeap L-S et al. Reciprocal transcriptional regulation of Pou5f1 and Sox2 via the Oct4/Sox2 complex in embryonic stem cells. Mol Cell Biol 2005; 25: 6031-6046.

17 Masui S, Nakatake Y, Toyooka Y, Shimosato D, Yagi R, Takahashi K et al. Pluripotency governed by Sox2 via regulation of Oct3/4 expression in mouse embryonic stem cells. Nat Cell Biol 2007; 9: 625-635.

18 Resnitzky D, Gossen M, Bujard H, Reed S. Acceleration of the G1/S phase transition by expression of cyclins D1 and $\mathrm{E}$ with an inducible system. Mol Cell Biol 1994; 14: 1669-1679.

19 Quelle D, Ashmun R, Shurtleff S, Kato J, Bar-Sagi D, Roussel M et al. Overexpression of mouse D-type cyclins accelerates G1 phase in rodent fibroblasts. Genes Dev 1993; 7: 1559-1571.
20 Ben-Shushan E, Thompson JR, Gudas LJ, Bergman Y. Rex-1, A gene encoding a transcription factor expressed in the early embryo, is regulated via Oct-3/4 and Oct- 6 binding to an octamer site and a novel protein, Rox-1, binding to an adjacent site. Mol Cell Biol 1998; 18: 1866-1878.

21 Hay DC, Sutherland L, Clark J, Burdon T. Oct-4 knockdown induces similar patterns of endoderm and trophoblast differentiation markers in human and mouse embryonic stem cells. Stem Cells 2004; 22: 225-235.

22 Niwa H, Miyazaki J-i, Smith AG. Quantitative expression of Oct-3/4 defines differentiation, dedifferentiation or self-renewal of ES cells. Nat Genet 2000; 24: 372-376.

23 Kopp JL, Ormsbee BD, Desler M, Rizzino A. Small increases in the level of Sox2 trigger the differentiation of mouse embryonic stem cells. Stem Cells 2008; 26: 903-911.

24 Tsai C-C, Su P-F, Huang Y-F, Yew T-L, Hung S-C. Oct4 and Nanog directly regulate Dnmt1 to maintain self-renewal and undifferentiated state in mesenchymal stem cells. Mol Cell 2012; 47: 169-182.

25 Rizzino A. The Sox2-Oct4 connection: critical players in a much larger interdependent network integrated at multiple levels. Stem Cells 2013; 31: 1033-1039.

26 Rodrigues M, Griffith LG, Wells A. Growth factor regulation of proliferation and survival of multipotential stromal cells. Stem Cell Res Ther 2010; 1: 32.

27 Jung S, Panchalingam KM, Rosenberg L, Behie LA. Ex vivo expansion of human mesenchymal stem cells in defined serum-free media. Stem Cells Int 2012; 2012: 123030.

28 Nombela-Arrieta CÃ Ś, Ritz J, Silberstein LE. The elusive nature and function of mesenchymal stem cells. Nat Rev Mol Cell Biol 2011; 12: 126-131.

(c) (1) $\odot \Theta$ This work is licensed under a Creative Commons Attribution-NonCommercial-NoDerivs 3.0 Unported License. The images or other third party material in this article are included in the article's Creative Commons license, unless indicated otherwise in the credit line; if the material is not included under the Creative Commons license, users will need to obtain permission from the license holder to reproduce the material. To view a copy of this license, visit http://creativecommons.org/licenses/by-nc-nd/3.0/ 